\title{
Novos registros e notas sobre distribuição geográfica de Trichoptera Kirby, 1813 (Insecta) do Estado de Mato Grosso, Brasil
}

\author{
Denis Silva Nogueira ${ }^{1}{ }^{3}$ \& Helena Soares Ramos Cabette ${ }^{2}$ \\ ${ }^{1}$ Programa de Pós-graduação em Ecologia e Conservação, \\ Universidade do Estado de Mato Grosso - UNEMAT, CP 08, CEP 78690-000, Nova Xavantina, MT, Brasil \\ ${ }^{2}$ Departamento de Ciências Biológicas, Universidade do Estado de Mato Grosso - UNEMAT, \\ CP 08, 78690-000, Nova Xavantina, MT, Brasil \\ ${ }^{3}$ Autor para correspondência: Denis Silva Nogueira, e-mail: dnogueira_bio@yahoo.com.br
}

\begin{abstract}
NOGUEIRA, D.S. \& CABETTE, H.S.R. New records and notes on geographic distribution of Trichoptera Kirby, 1813 (Insecta) from Mato Grosso State, Brazil. Biota Neotrop. 11(2): http://www.biotaneotropica.org. br/v11n2/en/abstract?article+bn03111022011
\end{abstract}

\begin{abstract}
Trichoptera comprise one of the most diverse and abundant group among aquatic insects in stream ecosystems around the world. Currently, about 500 species are known from Brazil, but only sixteen species have been recorded from the State of Mato Grosso. The present article provides a list of known species and new records of caddisflies collected in the eastern region over the past 10 years and incorporated in the entomological section of the Coleção Zoobotânica "James A. Ratter" of University of Mato Grosso State, Nova Xavantina (CZNX). Specimens were recorded from tributaries of the Rio Xingu and of the Rio das Mortes and from lakes and rivers of the Bananal floodplain. Altogether, 30 species are reported, being seven new records from Mato Grosso. Achoropsyche duodencimpunctata (Navás, 1916), Amazonatolica hamadae Holzenthal \& Pes, 2004, Nectopsyche nigricapilla (Navás, 1920), Nectopsyche quatourguttata (Navás, 1922), Macronema hageni Banks, 1924, Macrostemum santaeritae (Ulmer, 1905) and Cyrnellus fraternus (Banks, 1905) are new records from the State. Nectopsyche quatourguttata and N. nigricapilla configure the first records to Brazil. Additionally, Blepharopus diaphanus Kolenati, 1859, Macrostemum arcuatum (Erichson, 1848), Macrostemum ulmeri (Banks, 1913) are new regional records and provide additional material to species distribution from State.

Keywords: Amazônia-Cerrado transition, aquatic insects, inventories, aquatic ecosystems.
\end{abstract}

NOGUEIRA, D.S. \& CABETTE, H.S.R. Novos registros e notas sobre distribuição geográfica de Trichoptera Kirby, 1813 (Insecta) do Estado de Mato Grosso, Brasil. Biota Neotrop. 11(2): http://www.biotaneotropica. org.br/v11n2/pt/abstract?article+bn03111022011

Resumo: A ordem Trichoptera compreende uma das mais diversas e abundantes ordens dentre todos os grupos de insetos aquáticos encontrados em rios ao redor do mundo. Atualmente, cerca de 500 espécies são conhecidas no Brasil, mas apenas 16 espécies foram registradas para o Estado de Mato Grosso. O presente estudo apresenta uma lista das espécies conhecidas e novos registros, a partir de material coletado na região leste do estado nos últimos 10 anos e incorporado à seção entomológica da Coleção Zoobotânica "James A. Ratter" da Universidade do Estado de Mato Grosso, Nova Xavantina (CZNX). Foram registrados espécimes de tributários do Rio Xingu e do médio Rio das Mortes e em lagos e rios da planície de inundação do Bananal. Ao todo são reportadas 30 espécies, sendo sete novos registros para o Estado de Mato Grosso. Achoropsyche duodencimpunctata (Navás, 1916), Amazonatolica hamadae Holzenthal \& Pes, 2004, Nectopsyche nigricapilla (Navás, 1920), Nectopsyche quatourguttata (Navás, 1922), Macronema hageni Banks, 1924, Macrostemum santaeritae (Ulmer, 1905) e Cyrnellus fraternus (Banks, 1905) são novos registros para Mato Grosso. Nectopsyche quatourguttata e N. nigricapilla são registradas pela primeira vez para o Brasil. Adicionalmente, Blepharopus diaphanus Kolenati, 1859, Macrostemum arcuatum (Erichson, 1848) e Macrostemum ulmeri (Banks, 1913) configuram novos registros regionais e provêem material adicional para a distribuição das espécies no Estado.

Palavras-chave: transição Amazônia-Cerrado, insetos aquáticos, inventários, ecossistemas aquáticos. 


\section{Introdução}

A ordem Trichoptera é uma das mais abundantes e diversas, apresenta distribuição cosmopolita, não ocorrendo somente nas regiões polares e pequenas ilhas isoladas (De Moor \& Ivanov 2008). Extremamente importante para a dinâmica trófica dos ecossistemas aquáticos, sua contribuição em termos de biomassa tem sido enfatizada como uma das maiores dentre todos os insetos aquáticos (Wiggins 1996, Flint et al. 1999). Aproximadamente 13.500 espécies da ordem são atualmente conhecidas no mundo, sendo 2.530 na Região Neotropical (Morse 2011). Destas cerca de 500 são registradas no Brasil, principalmente para as regiões Sul, Sudeste e Norte (Paprocki et al. 2004, Dumas et al. 2010, Santos et al. 2010, Calor 2011).

Apesar do aumento substancial do número de espécies descritas e registradas para o Brasil nos últimos cinco anos (Blahnik et al. 2004, Blahnik 2005, Robertson \& Holzenthal 2005, 2006, Calor et al. 2006, Pes et al. 2008, Dumas \& Nessimian 2009a, b, Dumas et al. 2010, Nessimian \& Dumas 2010, Santos et al. 2010, Calor 2011, Quinteiro et al. 2011), poucos registros de ocorrência e descrições de espécies de Trichoptera têm sido acrescentadas às regiões Nordeste e Centro-oeste do país (Paprocki et al. 2004, Calor 2008a, b, Albino 2009), revelando muitas vezes uma distribuição disjunta em reflexo da carência de pesquisas nessas regiões. A despeito da falta de conhecimento da tricopterofauna, as estimativas indicam que o número de espécies possa chegar a mais de 50.000, mas apenas cerca de 20 a $25 \%$ da fauna mundial é atualmente conhecida (De Moor \& Ivanov 2008).

Diante de sua vasta rede hidrográfica e do aumento constante dos impactos das atividades antrópicas sobre seus mananciais e devido à importância desses organismos na manutenção da integridade ecológica dos ecossistemas aquáticos da região e no monitoramento da qualidade ambiental, o conhecimento da fauna de Trichoptera do Estado de Mato Grosso se faz essencial. Assim, nosso principal objetivo foi providenciar uma lista das espécies de Trichoptera para o Estado de Mato Grosso, adicionando novos registros e informações sobre suas distribuições, a partir de larvas e adultos coletados em rios da região Leste do estado.

\section{Material e Métodos}

Foram coletados representantes de Trichoptera de 40 locais, numa área de aproximadamente $600 \mathrm{~km}$ de extensão na região Leste de Mato Grosso, incluindo desde grandes lagos naturais conectados a rios da Planície de Inundação do Bananal, próximo a confluência do Rio das Mortes com o Rio Araguaia, a pequenos córregos com nascentes situadas nas encostas da Serra do Roncador e em afluentes do Rio Xingu mais ao Norte (Figura 1). Na Bacia do Rio Suiá-Miçú, afluente do Rio Xingu, foram identificadas larvas de Trichoptera coletadas em 11 locais, nos períodos de seca, chuva e vazante entre 2007 e 2008. Na Bacia do Rio das Mortes, os Trichoptera foram coletados em afluentes da Bacia do Rio Pindaíba, os quais correspondiam a córregos de nascente situados nas encostas da Serra do Roncador e rios variando da primeira à sexta ordem, totalizando 22 locais amostrados nos períodos de seca, chuva e vazante entre 2005 e 2008. Na Planície de Inundação do Bananal foram amostrados sete locais próximos à confluência do Rio das Mortes e Araguaia, que incluíram lagos, corixos e rios de grande porte (Figura 1, Tabela 1). O material encontra-se depositado e disponível na seção entomológica da Coleção Zoobotânica "James Alexander Ratter" (CZNX), na Universidade do Estado de Mato Grosso de Nova Xavantina.

As larvas foram identificadas em gênero com auxílio de chaves taxonômicas (Angrisano \& Korob 2001, Pes et al. 2005, Calor \& Froehlich 2008) e no nível de espécie através de descrições dos estágios imaturos (e.g. Holzenthal \& Pes 2004, Pes 2005, Pes et al. 2008, Albino 2009). Os adultos foram identificados com base em chaves e descrições de espécies (Flint 1978, Flint \& Wallace 1980, Flint et al. 1987) e revisadas por A.M.O. Pes (INPA). As espécies registradas, suas localidades de coleta, coordenadas geográficas latitudinais e longitudinais, altitude, datas são apresentados. Os respectivos números de tombamento dos espécimes na Coleção CZNX são apresentados entre colchetes e a forma de conservação entre parênteses. Comentários sobre a distribuição das espécies são apresentados para cada espécie. A lista das espécies e gêneros reportados para Mato Grosso, os novos registros de gêneros e espécies e a distribuição nos estados do Brasil são apresentados na Tabela 2.

\section{Resultados}

Foram identificadas 12 famílias, das quais apenas Hydropsychidae, Hydroptilidae e Philopotamidae eram formalmente reportadas para o Estado. Foram registradas as ocorrências de 30 gêneros, dos quais apenas nove eram conhecidos, e de 30 espécies sendo sete novos registros para Mato Grosso, três registros adicionais de distribuição de espécies já conhecidas para outras localidades no Estado e dois novos registros para o Brasil. As espécies Achoropsyche duodencimpunctata (Navás, 1916), Amazonatolica hamadae Holzenthal \& Pes, 2004, Nectopsyche nigricapilla (Navás, 1920), Nectopsyche quatourguttata (Navás, 1922), Macronema hageni Banks, 1924, Macrostemum santaeritae (Ulmer, 1905) e Cyrnellus fraternus (Banks, 1905) são registradas pela primeira vez para Mato Grosso. Nectopsyche quatourguttata e N. nigricapilla são novos registros para o Brasil, ampliando sua distribuição um pouco mais ao Leste da América do Sul. Adicionalmente, Blepharopus diaphanus Kolenati, 1859, Macrostemum arcuatum (Erichson, 1848), Macrostemum ulmeri (Banks, 1913) são registradas para outras localidades, ampliando sua distribuição no Estado.

Lista dos novos registros de espécies para Mato Grosso:

\section{HYDROPSYCHIDAE}

\section{Blepharopus diaphanus Kolenati, 1859}

Material examinado: 1 ㅇ [n ${ }^{\circ}$ 832] (em álcool), Brasil: Mato Grosso: Novo Santo Antônio, Lago Sucupira (P39), Parque Estadual do Araguaia,, 23/v/2004, S 12 19' 39,8' e O 50 55' 17,7', $207 \mathrm{~m}$ de altitude, col.: H.R.S. Cabette \& leg. Distribuição: Argentina, Venezuela e Brasil - RJ, SC, PR, MG e MT.

Comentários: esse gênero monotípico era previamente conhecido para outros países da América do Sul e para vários estados do Brasil (Flint et al. 1999, Blahnick et al. 2004, Paprocki et al. 2004), inclusive para Mato Grosso (Flint 1978). Na região Centro-oeste o único registro foi realizado na região de Nova Xavantina, mais precisamente no Rio Areões a cerca de $20 \mathrm{~km}$ da sede do município (Flint 1978). O registro no município de Novo Santo Antônio configura uma ampliação de sua distribuição a nordeste do estado, para a região da Planície de inundação do Bananal, provendo registro adicional a distribuição previamente conhecida da espécie no Estado.

\section{Macronema hageni Banks, 1924}

Material examinado: $1 \delta^{\hat{\gamma}}\left[\mathrm{n}^{\circ} 355\right]$ (a seco), Brasil: Mato Grosso: Cocalinho, Lago Olaria (P33), 11/viii/00, S 1304' 35, $0^{\circ}$ e O $51^{\circ} 05^{\prime}$ 50,2", $228 \mathrm{~m}$ de altitude, col. H.S.R. Cabette \& leg.

Distribuição: Argentina, Bolívia, Colômbia, Equador, Paraguai, Peru, Suriname, Venezuela e Brasil - AM, PA, MG e MT (novo registro).

Comentários: espécie registrada em vários países da América do Sul e para alguns estados da região Amazônica e no Estado de Minas 


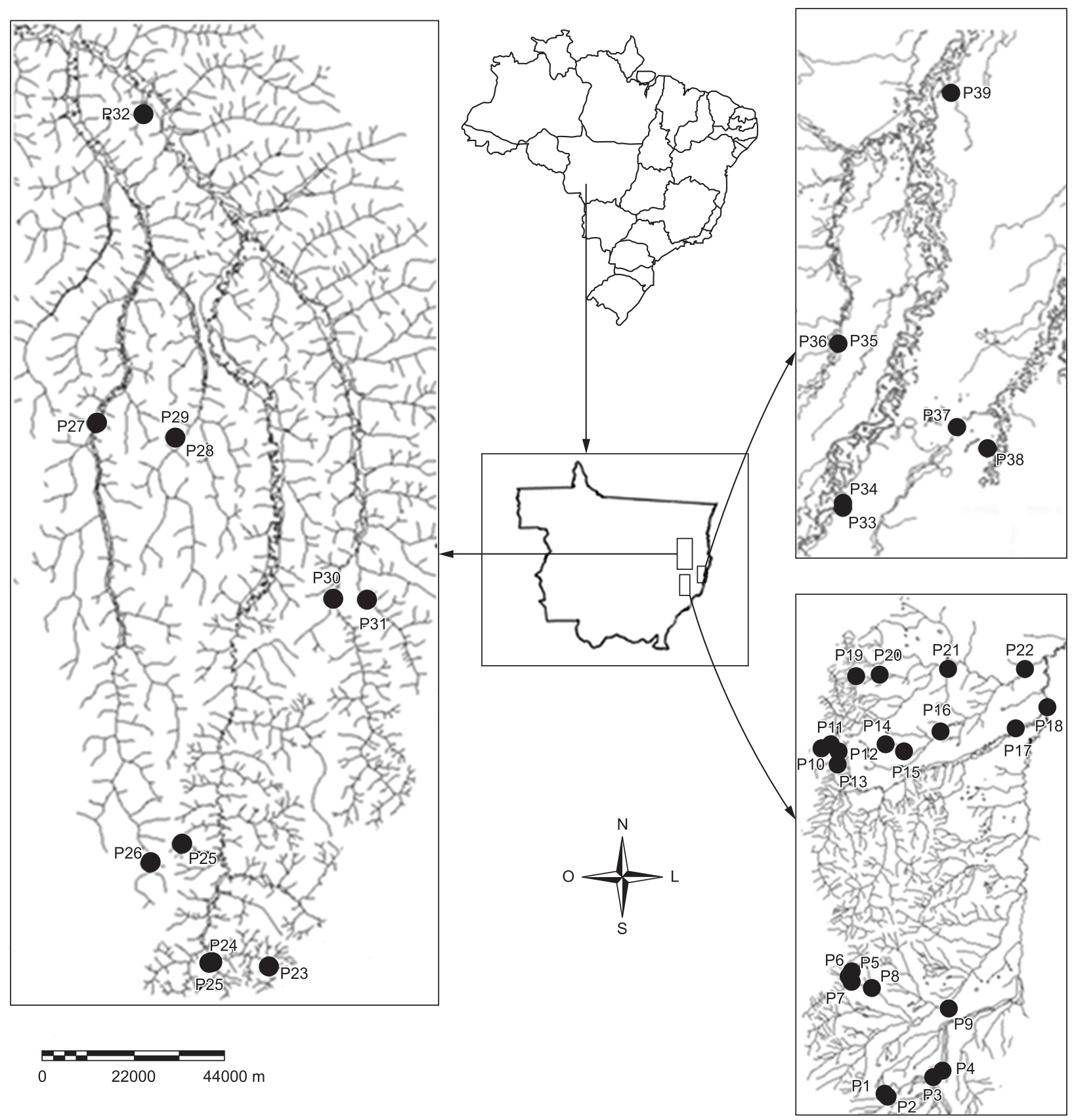

Figura 1. Mapa de distribuição dos locais de amostrados na região leste do Estado de Mato Grosso, Brasil. Os locais da Bacia do Rio Pindaíba estão representados no quadro à direita e abaixo, os locais do Pantanal Mortes - Araguaia estão representados no quadro à direita e acima e os locais da Bacia do Rio Suiá-Miçú estão representados à esquerda.

Figure 1. Map of distribution of samples sites from east of Mato Grosso State, Brazil. The samples sites of Rio Pindaíba Basin are represented in the picture to the right and below, the samples sites of Pantanal Mortes - Araguaia are represented in the picture to the right and above and the places of Rio Suiá-Miçú Basin are represented to the left.

Gerais (Flint et al. 1999, Paprocki et al. 2004), é pela primeira vez registrada em Mato Grosso, ampliando sua distribuição para a região Centro-oeste do Brasil.

\section{Macrostemum arcuatum (Erichson, 1848)}

Material examinado: 8 ㅇ [ $\mathrm{n}^{\circ}$ 819, 834] (em álcool), Brasil: Mato Grosso: Novo Santo Antônio, Lago Sucupira (P39), Parque Estadual do Araguaia, 24/v/04, S $12^{\circ} 19^{\prime} 39,8^{\prime \prime}$ e O 50 55' 17,7',

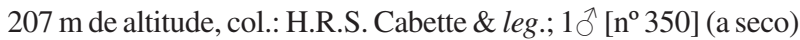
e 2 ㅇ $\left[\mathrm{n}^{\circ} 187,188\right]$ (em álcool), Brasil: Mato Grosso: Ribeirão
Cascalheira, Corixo Santa Cruz (P34), braço da margem direita do Rio das Mortes, 09 e 11/viii/00, S $12^{\circ} 58^{\prime}$ '20,8” e O 50 50' 36,6",

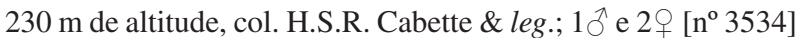
(em álcool), Brasil: Mato Grosso: Araguaiana, Rio Pindaíba em sexta ordem (P18), afluente do médio Rio das Mortes, 15/xi/05, S $14^{\circ} 54^{\prime} 10^{\prime \prime}$ e O 52 $00^{\prime} 21$ ”, $291 \mathrm{~m}$ de altitude, col.: H.S.R. Cabette \& leg.

Distribuição: Guiana, Peru, Suriname e Brasil - AM, PA e MT. Comentários: espécie de ocorrência conhecida para a região de abrangência da Amazônia Legal (Flint et al. 1999, Paprocki et al. 2004), 
Tabela 1. Lista dos sítios de amostragem, suas respectivas siglas e coordenadas geográficas dos tributários com novas ocorrências para o leste de Mato Grosso, Brasil, 1999/2008.

Table 1. List of samples sites, their respective abbreviaturies and geographical coordinates of tributaries with new occurrences recorded from east of Mato Grosso, Brazil, 1999/2008.

\begin{tabular}{|c|c|c|c|c|c|}
\hline Sítios & Hidrografia & Local & Siglas & Latitude S & Longitude W \\
\hline P5 & Bacia R. Corrente & C. Papagaio $1^{\mathrm{a}}$ ordem & CRP1 & $15^{\circ} 27^{\prime} 01^{\prime \prime}$ & $52^{\circ} 24^{\prime} 30^{\prime \prime}$ \\
\hline P6 & Bacia R. Corrente & C. Papagaio $2^{\mathrm{a}}$ ordem & CRP2 & $15^{\circ} 27^{\prime} 32^{\prime \prime}$ & $52^{\circ} 24^{\prime} 42^{\prime \prime}$ \\
\hline P7 & Bacia R. Corrente & C. Papagaio $3^{\mathrm{a}}$ ordem & CRP3 & $15^{\circ} 28^{\prime} 11^{\prime \prime}$ & $52^{\circ} 24^{\prime} 32^{\prime \prime}$ \\
\hline P8 & Bacia R. Corrente & C. Papagaio $4^{\mathrm{a}}$ ordem & CRP4 & $15^{\circ} 28^{\prime} 56^{\prime \prime}$ & $52^{\circ} 21^{\prime} 47^{\prime \prime}$ \\
\hline P1 & Bacia R. Corrente & C. Taquaral $1^{\mathrm{a}}$ ordem & CRT1 & $15^{\circ} 41^{\prime} 54^{\prime \prime}$ & $52^{\circ} 20^{\prime} 03^{\prime \prime}$ \\
\hline $\mathrm{P} 2$ & Bacia R. Corrente & C. Taquaral $2^{\mathrm{a}}$ ordem & CRT2 & $15^{\circ} 41^{\prime} 57^{\prime \prime}$ & $52^{\circ} 19^{\prime} 56^{\prime \prime}$ \\
\hline P3 & Bacia R. Corrente & C. Taquaral $3^{\mathrm{a}}$ ordem & CRT3 & $15^{\circ} 39^{\prime} 35^{\prime \prime}$ & $52^{\circ} 13^{\prime} 52^{\prime \prime}$ \\
\hline $\mathrm{P} 4$ & Bacia R. Corrente & C. Taquaral $4^{\mathrm{a}}$ ordem & CRT4 & $15^{\circ} 38^{\prime} 53^{\prime \prime}$ & $52^{\circ} 12^{\prime} 53^{\prime \prime}$ \\
\hline P9 & Bacia R. Corrente & Rio Corrente $5^{\mathrm{a}}$ ordem & RICO5 & $15^{\circ} 31^{\prime} 14^{\prime \prime}$ & $52^{\circ} 12^{\prime} 10^{\prime \prime}$ \\
\hline $\mathrm{P} 10$ & Bacia R. Pindaíba & C. Cachoeirinha $2^{\mathrm{a}}$ ordem & $\mathrm{CRC} 2$ & $14^{\circ} 50^{\prime} 50^{\prime \prime}$ & $52^{\circ} 24^{\prime} 22^{\prime \prime}$ \\
\hline $\mathrm{P} 11$ & Bacia R. Pindaíba & C. Cachoeirinha $3^{\mathrm{a}}$ ordem & CRC3 & $14^{\circ} 50^{\prime} 33^{\prime \prime}$ & $52^{\circ} 21^{\prime} 34^{\prime \prime}$ \\
\hline $\mathrm{P} 12$ & Bacia R. Pindaíba & C. Cachoeirinha $4^{\mathrm{a}}$ ordem & CRC4 & $14^{\circ} 49^{\prime} 45^{\prime \prime}$ & $52^{\circ} 12^{\prime} 55^{\prime \prime}$ \\
\hline P14 & Bacia R. Pindaíba & C. Caveira $1^{\mathrm{a}}$ ordem & CRCV1 & $14^{\circ} 59^{\prime} 06^{\prime \prime}$ & $52^{\circ} 20^{\prime} 29^{\prime \prime}$ \\
\hline $\mathrm{P} 15$ & Bacia R. Pindaíba & C. Caveira $2^{\mathrm{a}}$ ordem & CRCV2 & $14^{\circ} 59^{\prime} 53^{\prime \prime}$ & $52^{\circ} 18^{\prime} 17^{\prime \prime}$ \\
\hline P16 & Bacia R. Pindaíba & C. Caveira $3^{\mathrm{a}}$ ordem & CRCV3 & $14^{\circ} 57^{\prime} 28^{\prime \prime}$ & $52^{\circ} 13^{\prime} 43^{\prime \prime}$ \\
\hline $\mathrm{P} 10$ & Bacia R. Pindaíba & C. da Mata $1^{\mathrm{a}}$ ordem & CRM1 & $14^{\circ} 59^{\prime} 53^{\prime \prime}$ & $52^{\circ} 28^{\prime} 42^{\prime \prime}$ \\
\hline P11 & Bacia R. Pindaíba & C. da Mata $2^{\mathrm{a}}$ ordem & CRM2 & $14^{\circ} 59^{\prime} 18^{\prime \prime}$ & $52^{\circ} 27^{\prime} 30^{\prime \prime}$ \\
\hline $\mathrm{P} 12$ & Bacia R. Pindaíba & C. da Mata $3^{\mathrm{a}}$ ordem & CRM3 & $14^{\circ} 59^{\prime} 59^{\prime \prime}$ & $52^{\circ} 26^{\prime} 29^{\prime \prime}$ \\
\hline $\mathrm{P} 13$ & Bacia R. Pindaíba & C. da Mata $4^{\mathrm{a}}$ ordem & CRM4 & $15^{\circ} 01^{\prime} 32^{\prime \prime}$ & $52^{\circ} 26^{\prime} 29^{\prime \prime}$ \\
\hline P17 & Bacia R. Pindaíba & Rio Pindaíba $5^{\mathrm{a}}$ ordem & RIPI5 & $14^{\circ} 56^{\prime} 56^{\prime \prime}$ & $52^{\circ} 04^{\prime} 17^{\prime \prime}$ \\
\hline P18 & Bacia R. Pindaíba & Rio Pindaíba $6^{\mathrm{a}}$ ordem & RIPI6 & $14^{\circ} 54^{\prime} 10^{\prime \prime}$ & $52^{\circ} 00^{\prime} 21^{\prime \prime}$ \\
\hline P24 & Bacia R. Suiá-Miçú & Rio Piabanha & RIPB & $13^{\circ} 15^{\prime} 34.4^{\prime \prime}$ & $52^{\circ} 09^{\prime} 00.5^{\prime \prime}$ \\
\hline $\mathrm{P} 25$ & Bacia R. Suiá-Miçú & C. Lúcio & CRL & $13^{\circ} 05^{\prime} 34.5^{\prime \prime}$ & $52^{\circ} 15^{\prime} 16.9^{\prime \prime}$ \\
\hline P32 & Bacia R. Suiá-Miçú & C. Sucuri & CRSI & $11^{\circ} 49^{\prime} 50.7^{\prime \prime}$ & $52^{\circ} 17^{\prime} 02.2^{\prime \prime}$ \\
\hline $\mathrm{P} 25$ & Bacia R. Suiá-Miçú & C. Transição-Brejo & CRTB & $13^{\circ} 03^{\prime} 35.6^{\prime \prime}$ & $52^{\circ} 12^{\prime} 03.3^{\prime \prime}$ \\
\hline P31 & Bacia R. Suiá-Miçú & C.orrego Brejão & CRBJ & $12^{\circ} 38^{\prime} 32.3^{\prime \prime}$ & $51^{\circ} 53^{\prime} 20.6^{\prime \prime}$ \\
\hline P29 & Bacia R. Suiá-Miçú & R. Betis ponto 2 & RBT2 & $12^{\circ} 22^{\prime} 27.5^{\prime \prime}$ & $52^{\circ} 13^{\prime} 19.0^{\prime \prime}$ \\
\hline $\mathrm{P} 28$ & Bacia R. Suiá-Miçú & Rio Betis ponto 1 & RBT1 & $12^{\circ} 22^{\prime} 28.7^{\prime \prime}$ & $52^{\circ} 13^{\prime} 23.1^{\prime \prime}$ \\
\hline $\mathrm{P} 27$ & Bacia R. Suiá-Miçú & Rio Darro & RID & $12^{\circ} 21^{\prime} 12.3^{\prime \prime}$ & $52^{\circ} 21^{\prime} 27.4^{\prime \prime}$ \\
\hline $\mathrm{P} 23$ & Bacia R. Suiá-Miçú & Rio Suiá ponto 1 & RISU1 & $13^{\circ} 15^{\prime} 45.5^{\prime \prime}$ & $52^{\circ} 02^{\prime} 50.9^{\prime \prime}$ \\
\hline P24 & Bacia R. Suiá-Miçú & Rio Suiá ponto 2 & RISU2 & $13^{\circ} 15^{\prime} 24.3^{\prime \prime}$ & $52^{\circ} 08^{\prime} 44.5^{\prime \prime}$ \\
\hline P30 & Bacia R. Suiá-Miçú & Rio Suiazinho & RISUZ & $12^{\circ} 38^{\prime} 33.4^{\prime \prime}$ & $51^{\circ} 56^{\prime} 50.7^{\prime \prime}$ \\
\hline P38 & Pantanal do Araguaia & Lago Valdenor & LGV & $12^{\circ} 58^{\prime} 20.8^{\prime \prime}$ & $50^{\circ} 50^{\prime} 36.6^{\prime \prime}$ \\
\hline P33 & Pantanal do Araguaia & Lago Olaria & LGO & $13^{\circ} 04^{\prime} 35.0^{\prime \prime}$ & $51^{\circ} 05^{\prime} 50.2^{\prime \prime}$ \\
\hline P36 & Pantanal do Araguaia & Lago São João Grande & LGSJG & $12^{\circ} 58^{\prime} 20.8^{\prime \prime}$ & $50^{\circ} 50^{\prime} 36.6^{\prime \prime}$ \\
\hline P39 & Pantanal do Araguaia & Lago Sucupira & LGSU & $12^{\circ} 19^{\prime} 39.8^{\prime \prime}$ & $50^{\circ} 55^{\prime} 17.7^{\prime \prime}$ \\
\hline P34 & Pantanal do Araguaia & Corixo Sta. Cruz & COSC & $13^{\circ} 05^{\prime} 07.0^{\prime \prime}$ & $51^{\circ} 05^{\prime} 53.4^{\prime \prime}$ \\
\hline P37 & Pantanal do Araguaia & Rio Forquilha & RIF & $12^{\circ} 56^{\prime} 0.2^{\prime \prime}$ & $50^{\circ} 53^{\prime} 55.0^{\prime \prime}$ \\
\hline $\mathrm{P} 35$ & Pantanal do Araguaia & Rio São João Grande & RSJG & $12^{\circ} 47^{\prime} 12.2^{\prime \prime}$ & $51^{\circ} 06^{\prime} 42.7^{\prime \prime}$ \\
\hline
\end{tabular}

havia sido reportada para a região Noroeste de Mato Grosso, município de Aripuanã (Flint 1978). Esses registros ampliam a área de ocorrência da espécie em direção ao Leste do Estado.

Macrostemum santaeritae (Ulmer, 1905)

Material examinado: $1 ð$ e 99 [n $\left.n^{\circ} 3533\right]$ (em álcool), Brasil: Mato Grosso: Araguaiana, Rio Pindaíba em sexta ordem (P18), tributário da margem direita do médio Rio das Mortes, 15/xi/05, S 14 54' 10"e O 52 00' 21", $291 \mathrm{~m}$ de altitude, col.: H.S.R. Cabette \& leg.
Distribuição: Argentina e Brasil - AM, PA e MT (novo registro).

Comentários: espécie registrada para a Argentina e para a Amazônia brasileira é registrada pela primeira vez em Mato Grosso, o que amplia sua distribuição em direção à região Centro-oeste do Brasil.

\section{Macrostemum ulmeri (Banks, 1913)}

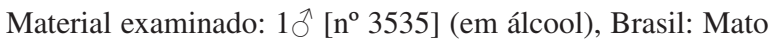
Grosso: Nova Xavantina, Córrego Caveira em segunda ordem 
Novos registros de Trichoptera (Insecta) para Mato Grosso, Brasil

Tabela 2. Lista das espécies de Trichoptera registradas para Mato Grosso, incluindo novos registros e distribuição nos estados do Brasil.

Table 2. List of Trichoptera species recorded from Mato Grosso, including new records and distribution by state of Brazil.

\begin{tabular}{|c|c|c|}
\hline Taxon & Bibliografia & Distribuição* \\
\hline \multicolumn{3}{|l|}{ Calamoceratidae } \\
\hline Phylloicus Müller, 1880 & Paprocki et al. (2004), Pes et al. (2005) & Novo registro em MT \\
\hline \multicolumn{3}{|l|}{ Ecnomidae } \\
\hline Austrotinodes Schmid, 1985 & Paprocki et al. (2004), Pes et al. (2005) & Novo registro em MT \\
\hline \multicolumn{3}{|l|}{ Glossosomatidae } \\
\hline Mortoniella (Ulmer, 1906) & Blahnik \& Holzenthal (2008) & Novo registro em MT \\
\hline Protoptila Banks, 1904 & Paprocki et al. (2004), Pes et al. (2005) & Novo registro em MT \\
\hline \multicolumn{3}{|l|}{ Helicopsychidae } \\
\hline Helicopsyche Siebold, 1859 & Paprocki et al. (2004), Pes et al. (2005) & Novo registro em MT \\
\hline \multicolumn{3}{|l|}{ Hydrobiosidae } \\
\hline Atopsyche Banks, 1905 & Paprocki et al. (2004), Pes et al. (2005) & Novo registro em MT \\
\hline \multicolumn{3}{|l|}{ Hydropsychidae } \\
\hline Blepharopus diaphanus Kolenati, 1859 & $\begin{array}{l}\text { Flint \& Wallace (1980), Paprocki et al. (2004), } \\
\text { Santos et al. (2010) }\end{array}$ & RJ, SC, PR, MG, AC e MT \\
\hline Leptonema amazonense Flint, 1978 & Flint (1978), Flint et al. (1987) & RR e MT, novo registro \\
\hline Leptonema aspersum (Ulmer, 1907) & Paprocki et al. (2004), Santos et al. (2010) & BA, MT e MS \\
\hline Leptonema crassum Ulmer, 1905 & Paprocki et al. (2004), Santos et al. (2010) & RR, MG, ES, SP, MT e GO \\
\hline Leptonema maculatum Mosely, 1933 & Paprocki et al. (2004), Flint et al. (1987) & AM, PA e MT \\
\hline Leptonema rostratum Flint, McAlpine \& Ross, 1987 & Paprocki et al. (2004), Santos et al. (2010) & AM, RR, PA e MT \\
\hline Leptonema sparsum (Ulmer), 1905 & Paprocki et al. (2004), Flint et al. (1987) & $\begin{array}{c}\text { RO, AM, PA, RJ, SP, SC, MT, } \\
\text { GO, DF, PR e MG }\end{array}$ \\
\hline Leptonema spinulum Flint, McAlpine \& Ross, 1987 & Dumas et al. (2010), Santos et al. (2010) & AM, DF, PA e MT \\
\hline
\end{tabular}

Macronema hageni Banks, 1924

Paprocki et al. (2004)

Macronema percitans Walker, 1860

Macronema pertyi Banks, 1924

Macrostemum arcuatum (Erichson, 1848)

Macrostemum hyalinum (Pictet, 1836)

Macrostemum santaeritae (Ulmer, 1905)

Macrostemum ulmeri (Banks, 1913)

Smicridea (Rhyacophylax) abrupta Flint, 1974

Smicridea (R.) appendiculata Flint, 1972

S. (R.) coronata Flint, 1980

S. (R.) ephippifer Flint, 1978

Smicridea (R.) mesembrina Navás 1918

S. (R.) palmar Sganga, 2005

Smicridea (Smicridea) palifera Flint, 1981

Smicridea (S.) truncata Flint, 1974

Synoestropsis furcata Flint, 1974

Synoestropsis grisoli Navás, 1924

Hydroptilidae

Bredinia espinosa Harris, Holzenthal \& Flint, 2002

Flintiella astilla Harris, Holzenthal \& Flint, 2002

Oxyethira Eaton, 1873

Leptoceridae

Achoropsyche duodecimpunctata (Navás, 1916)

Paprocki et al. (2004), Santos et al. (2010)

Paprocki et al. (2004), Santos et al. (2010)

Paprocki et al. (2004), Santos et al. (2010)

Paprocki et al. (2004)

Paprocki et al. (2004)

Paprocki et al. (2004)

Flint (1978), Paprocki et al. (2004), Santos et al. (2010)

J.L.D. Albino et al. (dados não publicados)

J.L.D. Albino et al. (dados não publicados)

J.L.D. Albino et al. (dados não publicados)

J.L.D. Albino et al. (dados não publicados)

J.L.D. Albino et al. (dados não publicados)

Paprocki et al. (2004)

Flint (1974), Paprocki et al. (2004), Pes et al. (2008)

Calor (2008b), Santos et al. (2010)

Paprocki et al. (2004), Santos et al. (2010)

Paprocki et al. (2004), Santos et al. (2010)

Santos et al. (2010)

Paprocki et al. (2004), Pes et al. (2005)

Holzenthal (1984)

$\mathrm{AM}, \mathrm{PA}, \mathrm{MG}$ e MT, novo registro

AM, PA e MT

MT

AM, PA e MT

MT, novo registro

MT, novo registro

MT, novo registro

MT e AM

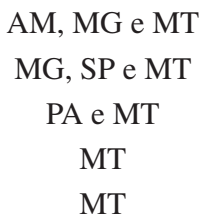

RJ, MT, novo registro

AM, PA e MT, novo registro

PA, MT

AM, PA, MG e MT

RO e MT

BA, PR, SP e MT

$\mathrm{MT}$, novo registro

AM, RR, PA, ES, SC, PR, MG, $\mathrm{SP}$ e MT, novo registro AM, BA, RO e MT, novo registro 
Tabela 2. Continuação...

\begin{tabular}{|c|c|c|}
\hline Taxon & Bibliografia & Distribuição* \\
\hline Amphoropsyche Holzenthal, 1985 & Holzenthal (1985) & MT, novo registro \\
\hline Nectopsyche nigricapilla (Navás, 1920) & Flint et al. (1999) & MT, novo registro para o Brasil \\
\hline Nectopsyche quatourguttata (Navás, 1922) & Flint et al. (1999) & MT, novo registro para o Brasil \\
\hline Notalina Mosely, 1936 & $\begin{array}{l}\text { Paprocki et al. (2004), Holzenthal (1986), } \\
\text { Calor \& Froehlich (2008) }\end{array}$ & MT, novo registro \\
\hline Oecetis McLachlan, 1877 & Paprocki et al. (2004), Pes et al. (2005) & MT, novo registro \\
\hline Triplectides Kolenati, 1859 & $\begin{array}{c}\text { Paprocki et al. (2004), Pes et al. (2005), } \\
\text { Holzenthal (1988) }\end{array}$ & \\
\hline \multicolumn{3}{|l|}{ Odontoceridae } \\
\hline Marilia Müller, 1880 & Paprocki et al. (2004), Pes et al. (2005) & MT, novo registro \\
\hline \multicolumn{3}{|l|}{ Philopotamidae } \\
\hline Chimarra (Curgia) conica Flint, 1998 & Paprocki et al. (2004), Santos et al. (2010) & $\mathrm{CE}, \mathrm{GO}, \mathrm{MG}, \mathrm{MT}, \mathrm{RJ}, \mathrm{RO}$ e SC \\
\hline Wormaldia McLachlan, 1865 & Paprocki et al. (2004), Pes et al. (2005) & MT, novo registro \\
\hline \multicolumn{3}{|l|}{ Polycentropodidae } \\
\hline Cernotina Ross, 1938 & Paprocki et al. (2004), Pes et al. (2005) & MT, novo registro \\
\hline Cyrnellus fraternus (Banks, 1905) & Paprocki et al. (2004) & $\begin{array}{l}\mathrm{AM}, \mathrm{PA}, \mathrm{MG}, \mathrm{PR}, \mathrm{SC} \text { e } \mathrm{MT}, \\
\text { novo registro }\end{array}$ \\
\hline Polycentropus Curtis, 1835 & Paprocki et al. (2004) & MT, novo registro \\
\hline Polyplectropus Ulmer, 1905 & Paprocki et al. (2004), Pes et al. (2005) & MT, novo registro \\
\hline \multicolumn{3}{|l|}{ Xiphocentronidae } \\
\hline Xiphocentron Brauer, 1870 & Paprocki et al. (2004) & MT, novo registro \\
\hline
\end{tabular}

*AC = Acre; $\mathrm{AM}=$ Amazonas; $\mathrm{BA}=$ Bahia; $\mathrm{DF}=$ Distrito Federal; $\mathrm{GO}=$ Goiás; ES = Espírito Santo; MG = Minas Gerais; MT = Mato Grosso;

$\mathrm{PA}=$ Pará; $\mathrm{PR}=$ Paraná; RJ = Rio de Janeiro; RR = Roraima; $\mathrm{SC}=$ Santa Catarina; e SP = São Paulo.

(P15), tributário da Bacia do Rio Pindaíba, 06/v/08, S 14 59' 53" e O $52^{\circ} 18^{\prime}$ '17', 328 m de altitude, col.: H. S.R. Cabette \& leg.

Distribuição: Colômbia, Costa Rica, Equador, Honduras, Panamá, Peru, Suriname e Brasil - AM, PA, RO, MG e MT.

Comentários: espécie de ampla distribuição em toda a região neotropical (Flint et al. 1999), ocorre em vários estados brasileiros principalmente da região Norte e Sudeste (Paprocki et al. 2004). Esta espécie foi pela primeira vez registrada em Mato Grosso a partir de uma fêmea (Flint 1978), contudo, apesar das coordenadas geográficas apresentadas corresponderem a região leste de Mato Grosso, referências quanto as localidades não foram especificadas. Sendo assim este registro configura material adicional ao conhecimento da distribuição da espécies no Estado.

\section{LEPTOCERIDAE}

Achoropsyche duodencimpunctata (Navás, 1916)

Material examinado: $122 \jmath^{\text {e } 46}$ \% [ $\mathrm{n}^{\circ} 253,254,256,257,258$, 259, 260, 262, 267, 383, 386] (em álcool) e [n 105, 106, 107, 108, 109, 110, 111, 112, 113, 114] (a seco), Brasil: Mato Grosso: Ribeirão Cascalheira, Corixo Santa Cruz (P34), braço direito do Rio das Mortes, 09-10/viii/2000 e 15/v/2001, S $12^{\circ}$ 58' 20,8" e O 50 50' 36,6”, $230 \mathrm{~m}$ de altitude, col.: H.S.R Cabette \& leg.;

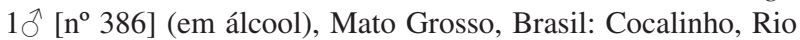
Forquilha (P37), 26/vi/2000, S $12^{\circ} 56^{\prime}$ 0,2" e O 50' 53' 55,0", altitude de 232 m, col.: H.S.R Cabette \& leg.; $4 \hat{\jmath}$ e 90 [ [ ${ }^{\circ} 260$ ] (em álcool), Mato Grosso, Brasil: Novo Santo Antonio, Rio São João Grande, (P36), 29/vi/2000, S 1247' 12,2”' e O 51 06' 42,7”, $221 \mathrm{~m}$ de altitude, col.: H.S.R. Cabette \& leg.

Distribuição: Argentina, Bolívia, Colômbia, Equador, Guiana, Paraguai, Peru, Suriname, Uruguai, Venezuela e Brasil-AM, RO, PA, ES, SC, PR, MG, SP e MT (novo registro).
Comentários: essa espécie foi descrita no gênero Setodes por Navás (1916) e posteriormente deslocada para Brachysetodes por Flint (1972). Holzenthal (1984) revisou o gênero Brachysetodes e criou uma nova tribo, Achoropsychini, transferindo essa espécie para o gênero monotípico Achoropsyche. Espécie de ampla distribuição na América do Sul (Holzenthal 1984, Flint et al. 1999), ocorre em vários estados da região Norte, Sul e Sudeste do Brasil (Paprocki et al. 2004). Neste estudo, adultos foram coletados próximos a lagos e rios de grande porte, corroborando informações da descrição (Holzenthal 1984), sendo pela primeira vez registrada em Mato Grosso, ampliando sua distribuição para a região Centro-oeste do Brasil.

Amazonatolica hamadae Holzenthal \& Pes, 2004

Material examinado: 7 larvas [ $\mathrm{n}^{\circ} 3097,3100,3113,3114,3115$, 3141] (em álcool), Brasil: Mato Grosso: Querência, Córrego Lúcio na segunda ordem (P25), afluente do Rio Darro, 19/xii/07, 23/v/07 e 23/v/08, S $13^{\circ} 05^{\prime} 34,5^{\prime \prime}$ e O $52^{\circ} 15^{\prime} 16,9^{\prime}$ ', $355 \mathrm{~m}$ de altitude, col.: H.S.R. Cabette \& leg.

Distribuição: Brasil - AM, BA, RO e MT (novo registro).

Comentário: espécie de distribuição aparentemente disjunta no Brasil, não havia sido formalmente registrada para nenhuma outra localidade desde sua descrição. Essa espécie foi encontrada em um riacho de pequeno porte preservado e de águas levemente ácidas da transição Cerrado-Floresta Amazônica, corroborando as informações da descrição (Holzenthal \& Pes 2004), sendo este seu primeiro registro em Mato Grosso, o que amplia sua distribuição em direção a borda Sul - Amazônica a nordeste do Estado.

\section{Nectopsyche quatourguttata (Navás, 1922)}

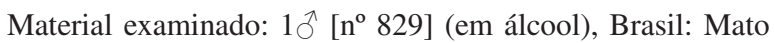
Grosso: Novo Santo Antônio, Rio das Mortes, Corixo Santa Cruz (braço do Rio das Mortes, Mato Grosso, Brasil), 23/v/04, 
S $13^{\circ} 05^{\prime} 07,0^{\prime \prime}$ e $\mathrm{O} 51^{\circ} 05^{\prime}$ 53,4”, $232 \mathrm{~m}$ de altitude, col.: H.S.R Cabette \& leg.; $1 \delta^{\widehat{O}}$ e 2 ㅇ [n ${ }^{\circ}$ 817] (em álcool), Brasil: Mato Grosso: Novo Santo Antônio, Lago São João Grande (P36), 21/v/04, S $12^{\circ} 58^{\prime} 20,8^{\prime \prime}$ e O 50 50' 36,6", altitude de $230 \mathrm{~m}$, col.: H.S.R. Cabette \& leg.

Distribuição: Bolívia, Peru, Guiana, Suriname e Brasil - MT (novo registro).

Comentário: os exemplares desta espécie haviam sido previamente reportados para a Bolívia por Navás como Leptocella Navás, 1922. Schmid (1949) transferiu estes exemplares para o gênero Nectopsyche Müller, 1879 e registros adicionais foram providos para o Peru, Guiana e Suriname (Flint 1974, 1996, Flint et al. 1999). O registro em Mato Grosso é o primeiro da espécie para o Brasil, ampliando sua distribuição em direção leste na América do Sul.

\section{Nectopsyche nigricapilla (Navás, 1922)}

Material examinado: $1 \hat{\jmath}$ e 1 ㅇ [no 255, 263] (em álcool), Brasil: Mato Grosso: Cocalinho, Rio Forquilha (P37), 13/v/01, S $12^{\circ} 56^{\prime} 00,2^{\prime \prime}$ e O 50 53' 55,0”, $232 \mathrm{~m}$ de altitude, col.: H.S.R. Cabette \& leg.

Distribuição: Paraguai, Argentina, Colômbia e Brasil - MT (novo registro).

Comentário: essa espécie foi descrita por Navás em Leptocella, a partir de exemplares coletados no Paraguai. Schmid (1949) a transferiu para Nectopsyche e Flint (1972) proveu registros distribucionais e sinonímia de Leptocella ornatta (Navás, 1933). O registro em Mato Grosso é o primeiro para o Brasil, ampliando sua distribuição em direção a leste na América do Sul.

\section{POLYCENTROPODIDAE}

\section{Cyrnellus fraternus (Banks, 1905)}

Material examinado: $4 \hat{\jmath}$ e 11 우 [n $\left.\mathrm{n}^{\circ} 820,824,827,831\right]$ (em álcool), Brasil: Mato Grosso: Novo Santo Antônio, Lago Sucupira (P39), Parque Estadual do Araguaia, 23-24/v/2004, 21/vi/2004, S $12^{\circ} 19^{\prime} 39,8^{\prime \prime}$ e O $50^{\circ} 55^{\prime} 17,7^{\prime}$ ', 207 m de altitude, col.: H. S.R. Cabette \& leg.

Distribuição: Costa Rica, Equador, El Salvador, Estados Unidos, México, Nicarágua, Panamá, Paraguai, Suriname, Uruguai, Venezuela e Brasil - AM, PA, MG, PR, SC e MT (novo registro).

Comentários: esta espécie apresenta ampla distribuição nas Américas (Flint et al. 1999) ocorrendo em vários Estados do Brasil (Paprocki et al. 2004). Sua ocorrência era desconhecida na região Centro-oeste do Brasil, sendo o primeiro registro da espécie no Estado.

\section{Discussão}

Paprocki et al. (2004) registraram 378 espécies para o país, mas apenas oito para Mato Grosso: Leptonema crassum Ulmer, 1905, L. sparsum Ulmer, 1905, L. rostratum Flint McAlpine \& Ross, 1987, L. spinulum Flint McAlpine \& Ross, 1987, Macronema pertyi Banks, 1924, M. percitans Walquer, 1860 (Hydropsychidae), Bredinia espinosa Harris et al., 2002 (Hydroptilidae) e Chimarra (Curgia) conica Flint, 1983 (Philopotamidae). Contudo, estes autores não incluíram em seus registros espécies previamente reportadas para o Estado por Flint (1978): L. aspersum (Ulmer, 1907), L. maculatum (Mosely, 1933), Smicridea abrupta Flint 1974, Synoestropsis furcata Flint, 1974 e Synoestropsis grisoli Navás, 1924, incluídas na lista das espécies para os Estados do Brasil (Santos et al. 2010), o que duplicou a lista da fauna conhecida para 16 espécies.

Recentemente, Calor (2008b) registrou a ocorrência de Synoestropsis furcata Flint, 1974 na região de Nova Xavantina e descreveu larvas obtidas no Rio Suiá-Miçú, no município de Ribeirão Cascalheira, Leste de Mato Grosso. Analisando a fauna de Smicridea de tributários da Bacia do Rio Pindaíba, Albino (2009) adiciona oito espécies para o Estado, sendo quatro espécies novas e ainda não descritas (não incluídas na lista) e quatro novos registros. Calor (inf. pessoal) relata ainda, a existência de espécies novas de Triplectides e de uma possível espécie nova de Oecetis para o Estado, contudo esse material está ainda em análise. Somando as espécies registradas nos inventários da fauna de Trichoptera com informações para Mato Grosso (Flint 1978, Paprocki et al. 2004, Calor 2008b, Albino 2009), aos 10 registros do presente estudo, tem-se um total de 30 espécies conhecidas no Estado.

Para o Brasil, o número atualizado de espécies é aproximadamente 520, mas considerando o número de espécies depositadas em museus do Brasil e do exterior (Calor $2009 \approx 300$ espécies), ainda por serem descritas, espera-se que esse montante possa aumentar consideravelmente. Além disso, boa parte dos Trichoptera amostrados em estudos prévios na região leste de Mato Grosso se restringe a larvas não associadas a adultos e permanece ainda sem identificação no nível específico. Estas se encontram morfoespeciadas na coleção CZNX, totalizando 109 morfoespécies até então (Nogueira 2011), revelando que o conhecimento taxonômico da fauna de Trichoptera é extremamente precário e merecedor de atenção em especial no Cerrado e na região de transição Cerrado-Floresta Amazônica. Os ambientes aquáticos são extremamente variados no Mato Grosso, onde três importantes biomas brasileiros estão representados (Cerrado, Floresta Amazônica e Pantanal), o que faz estes estudos ainda mais atrativos.

Uma das principais lacunas de conhecimento acerca da ordem é a carência de informações taxonômicas para larvas, o que impossibilita as atividades de biomonitoramento e escolha de espécies indicadoras de qualidade ambiental. A distribuição fragmentada de muitas espécies conhecidas para o Brasil tem se mostrado muito mais um artefato da carência de estudos, uma vez que muitas delas apresentam registros somente para sua localidade-tipo (Dumas et al. 2010). Conclusões a respeito dos padrões biogeográficos de grupos previamente tidos como endêmicos e de distribuição restrita podem estar representando na realidade a deficiência de estudos com a fauna da região como verificado para outros estados brasileiros (Blahnick et al. 2004).

A fauna de Trichoptera no Estado de Mato Grosso é ainda pobremente conhecida, neste sentido, a criação de larvas para associação aos adultos juntamente com estudos de levantamentos regionais dos adultos se mostram promissores para o melhor entendimento da taxonomia, biogeografia e ecologia destes insetos.

\section{Agradecimentos}

Nós agradecemos Dra . Gisele L. Almeida $(M N)$ e à Dra . Ana Maria O. Pes (INPA) pelo suporte na identificação de vários exemplares de Trichoptera, ao Dr. Frederico F. Salles, ao Dr. Jorge Luiz Nessimian e a Dra. A.M.O. Pes (INPA) pela revisão de uma versão prévia deste manuscrito e a dois revisores anônimos pelas ricas sugestões. Ao CNPq, Proc. n ${ }^{\circ}$ 520345/99-1, PROBIO/MMA/, Proc. nº 680020/02-0, à FAPEMAT, Proc. nº 098/2004, à FAPEMAT, Proc. nº 0907/2006 e ao CNPq, Proc. $n^{\circ} 520268 / 2005-9$ pelo fomento aos projetos. O primeiro autor é grato pela bolsa de Mestrado CAPES e ao Laboratório de Entomologia de Nova Xavantina pelo suporte laboratorial e auxílio em campo.

\section{Referências Bibliográficas}

ALBINO, J.L.D. 2009. Taxonomia do gênero Smicridea McLachlan (Trichoptera: Hydropsychidae) de três estado da Amazônia. Dissertação de Mestrado, Universidade Federal do Amazonas, Manaus. 
ANGRISANO, E.B. \& KOROB, P.G. 2001. Trichoptera. In Guia para la identificacion de los artrópodos bentônicos sudamericanos (H. R. Fernández \& E. Domínguez, eds.). Universidade Nacional de Tucamán, Tucamám.

BLAHNIK, R.J. 2005. Alterosa, a new caddisfly genus from Brazil (Trichoptera: Philopotamidae). Zootaxa. 991: 1-60.

BLAHNIK, R.J. 2002. Systematics of Otarrha, a new Neotropical subgenus of Chimarra (Trichoptera: Philopotamidae). Syst. Entomol. 27:65-130. http://dx.doi.org/10.1046/j.0307-6970.2001.00166.x

BLAHNIK, R.J. \& HOLZENTHAL, R.W. 2008. Revision of the Mexican and Central American species of Mortoniella (Trichoptera: Glossosomatidae: Protoptilinae). Zootaxa. 1711:1-72.

BLAHNIK, R.J., PAPROCKI, H. \& HOLZENTHAL, R.W. 2004. New distribution and species records of Trichoptera from southern and southeastern Brazil. Biota Neotrop. 4(1):1-6. http://www.biotaneotropica. org.br/v4n1/pt/abstract?inventory+BN01304012004 (ultimo acesso em 22/09/2010).

CALOR, A.R. 2008a. A new species of Notalina Mosely, 1936 (Trichoptera: Leptoceridae) from Chapada dos Veadeiros National Park, Goiás State, Brazil. Biota Neotrop. 8(3):175-178. http://www.biotaneotropica.org.br/ v8n3/en/abstract?article+bn02108032008 (ultimo acesso em 22/09/2010).

CALOR, A.R. 2008b. Description of the larvae of Synoestropsis furcata Flint, 1974 (Trichoptera: Hydropsychidae) from central-western region, Brazil. Aquat. Insects. 30(4):319-325. http://dx.doi. org/10.1080/01650420802334079

CALOR, A.R. 2009. Considerações Acerca da Filogenia de Trichoptera Kirby 1813: da Análise dos Dados para as Hipóteses ou dos Cenários para os Dados. EntomoBrasilis. 2: 1-10.

CALOR, A.R. 2011. Checklist dos Trichoptera (Insecta) do Estado de São Paulo, Brasil. Biota Neotrop. 11(1):prelo.

CALOR, A.R. \& FROEHLICH, C.G. 2008. Description of the immature stages of Notalina morsei HOLZENTHAL, 1986 (Trichoptera: Leptoceridae) and an updated key to larvae of Neotropical Leptoceridae genera. Zootaxa. 1779:45-54.

CALOR, A.R., HOLZENTHAL, R.W. \& D.S. AMORIM. 2006. Phylogenetic analysis of Notalina (Neonotalina) Holzenthal (Trichoptera: Leptoceridae), with the description of two new species from southeastern Brazil. Zootaxa. 1131:33-48.

DE MOOR, F.C. \& IVANOV, V.D. 2008. Global diversity of caddisflies (Trichoptera: Insecta) in freshwater. Hydrobiologia. 595:393-407. http:// dx.doi.org/10.1007/s10750-007-9113-2

DUMAS, L.L. \& NESSIMIAN, J.L. 2009a. A new species of Leptonema Guérin (Trichoptera: Hydropsychidae) from southeastern Brazil. Zootaxa. 2245:65-68.

DUMAS, L.L. \& NESSIMIAN, J.L. 2009b. Description of two new species of Marilia Müller (Trichoptera, Odontoceridae) from southeastern Brazil, including the description of the female of Marilia major Müller. Rer. Bras. Entomol. 53(3):344-348. http://dx.doi.org/10.1590/S008556262009000300006

DUMAS, L.L., SANTOS, A.P.M., JARDIM, G.A., FERREIRA-JUNIOR, N. \& NESSIMIAN, J.L. 2010. Insecta, Trichoptera: new records from Brazil and other distributional notes. Checklist. 6(1):7-9.

FLINT JUNIOR, O.S. 1972. Studies of Neotropical Caddisflies, XIV: on a collection from northen Argentina. Proc. Biol. Soc. Washing. 75:219.

FLINT JUNIOR, O.S. 1974. Studies of Neotropical Caddisflies, XV: the Trichoptera of Surinam. Stud. Fauna Suri. Guianas. 14(55):1-151.
FLINT JUNIOR, O.S. 1978. Studies of Neotropical Caddisflies, XXII: Hydropsychidae of Amazon Basin (Trichoptera). Amazoniana. 6(3):373-421.

FLINT JUNIOR, O.S. 1996. The Trichoptera colleted on the expeditions to Parque Manu, Madre de Dios, Peru. 369-430pp. In Manu: The biodiversity of southeastern Peru (D.E. Wilson \& A. Sandoval, eds.). Smithsonian Institution Press, Washington.

FLINT JUNIOR, O.S., HOLZENTHAL, R.W. \& HARRIS, S.C. 1999. Catalog of the Neotropical Caddisflies (Insecta: Trichoptera). Ohio Biological Survey, Ohio, 239p.

FLINT JUNIOR, O.S., MCALPINE, J.F. \& ROSS, H.H. 1987. A revision of the Genus Leptonema Guérin (Trichoptera: Hydropsychidae: Macronematinae). Smith. Contrib. Zool. 450:1-193. http://dx.doi. org/10.5479/si.00810282.450

FLINT JUNIOR, O.S. \& WALLACE, J.B. 1980. Studies of Neotropical Caddisflies, XXV: The immature stages of Blepharopus diaphanus and Leptonema columbianum (Trichoptera: Hydropsychidae). Proc. Biol. Soc. Washing. 93(1):178-193.

HOLZENTHAL, R.W. 1984. Studies in Neotropical Leptoceridae (Trichoptera) 1: Achoropsyche, a new genus. Proc. Int. Symp. Trichoptera. 4:181-184.

HOLZENTHAL, R.W. 1985. Studies in Neotropical Leptoceridae (Trichoptera), II: Amphoropsyche, a new genus and species of Leptocerinae from northern South America. International Journal of Entomology. 27: 254-269.

HOLZENTHAL, R.W. 1986. The Neotropical species of Notalina, a southern group of long-horned caddisflies (Trichoptera: Leptoceridae). Syst. Entomol. 11:61-73. http://dx.doi.org/10.1111/j.1365-3113.1986. tb00165.x

HOLZENTHAL, R.W. 1988. Systematics of Neotropical Triplectides (Trichoptera: Leptoceridae). Ann. Entomol. Soc. Am. 81(2):187-208.

HOLZENTHAL, R.W. \& PES, A.M.O. 2004. A new genus of longhorned caddisfly from the Amazon basin (Trichoptera: Leptoceridae: Grumichellini). Zootaxa. 621:1-16.

MORSE, J.C., (ed.). 2011. Trichoptera World Checklist. http://entweb. clemson.edu/database/trichopt/index.htm (acessado em 22/02/2011)

NESSIMIAN, J.L. \& DUMAS, L.L. 2010. Description of the immature stages of Leptonema tridens (Insecta: Trichoptera: Hydropsychidae) from southeastern Brazil with notes on its biology. Zoologia. 27:465-471.

PAPROCKI, H., HOLZENTHAL, R.W. \& BLAHNIK, R.J. 2004. Checklist of the Trichoptera (Insecta) of Brazil I. Biota Neotrop. 4(1):2-22. http://www. biotaneotropica.org.br/v4n1/pt/abstract?inventory+BN01204012004 (ultimo acesso em 22/09/2010).

PES, A.M.O. 2005. Taxonomia, estrutura e riqueza das comunidades de larvas e pupas de Trichoptera (Insecta), em igarapés na Amazônia Central. Tese doutorado, Universidade Federal do Amazonas, Manaus.

PES, A.M.O., HAMADA, N. \& NESSIMIAN, J.L. 2005. Chaves de identificação de larvas para famílias e gêneros de Trichoptera (Insecta) da Amazônia Central, Brasil. Rev. Bras. Entomol. 49:181-204. http:// dx.doi.org/10.1590/S0085-56262005000200002

PES, A.M.O., HAMADA, N. \& SOARES, C.C. 2008. Description of the last-instar larva and pupa and the bionomics of Smicridea (Smicridea) truncata Flint (Trichoptera: Hydropsychidae) in Central Amazonia, Brazil. Zootaxa. 1732:56-60.

QUINTEIRO, F.B., CALOR, A.R. \& FROEHLICH, C.G. 2011. A new species of Phylloicus Müller, 1880 (Trichoptera: Calamoceratidae), from southeastern Brazil, including descriptions of larval and pupal stages. Zootaxa. 2748:38-46. 
ROBERTSON, D.R. \& HOLZENTHAL, R.W. 2005. The Neotropical caddisfly genus Tolhuaca (Trichoptera: Glossosomatidae). Zootaxa. 1063:53-68.

ROBERTSON, D.R. \& HOLZENTHAL, R.W. 2006. The Neotropical caddisfly genus Canoptila (Trichoptera: Glossosomatidae). Zootaxa. 1272:45-59.
SANTOS, A.P.M., DUMAS, L.L., JARDIM, G.A. \& NESSIMIAN, J.L. 2010. Brazilian Caddisflies: Checklists and Bibliography. https://sites.google. com/site/braziliancaddisflies (ultimo acesso em 20/09/2010)

SCHMID, F. 1949. Les Trichoptères de la Collection Navás. Eos. 25:305-426.

WIGGINS, G.B. 1996. Larvae of the North American Caddisfly Genera (Trichoptera). University of Toronto Press, Toronto, 457p.

Recebido em 30/09/2010 Versão reformulada recebida em 01/05/2011

Publicado em 08/06/2011 